\title{
A Note on Optimal Allocation Mechanisms*
}

\author{
Nicolás Figueroa, Universidad de Chile-CEA ${ }^{\dagger} \quad$ Vasiliki Skreta, NYU-STern ${ }^{\ddagger}$
}

April 1, 2008

\begin{abstract}
This paper studies revenue-maximizing allocation mechanisms for multiple goods where the buyer's utility can depend non-linearly in his type. We point out that despite strictly increasing virtual utilities, the allocation rule obtained via pointwise optimization may fail to be increasing and thus it may violate incentive compatibility. More importantly, the revenue maximizing allocation may involve randomizations between different allocations. Keywords: mechanism design, optimal auctions, bunching. JEL Classification Codes: C72, D44, D82.
\end{abstract}

The literature on revenue-maximizing mechanisms is not only central to auction theory, but to economic theory in general. In the seminal contributions of Myerson (1981) and Riley and Samuelson (1981) two key features appear: first, that revenue-maximizing mechanisms are deterministic, in the sense that either a good is obtained with probability one by a buyer, or it is kept by the seller, and, second, that the problem can be solved through pointwise maximization if virtual utilities (utility minus information rents) are strictly monotonic in a buyer's type.

Our findings contrast both fundamental features of those papers. We present an example with two goods and where the buyer's utility depends non-linearly on his type. We establish that the allocation rule obtained via pointwise optimization may not be increasing and thus it may violate incentive compatibility, even if virtual utilities are strictly monotonic in type. When the monotonicity constraint binds despite monotone virtual utilities, previous methods of solving for a revenue-maximizing mechanism fail because they rely on making virtual utilities monotonic ("ironing"). We propose a solution method and with its help show that an optimal mechanism involves randomizations over different goods. In some sense, a certain range of consumer types are offered a random "bundle" of goods. Our method has the advantage that it does not require mechanisms to be differentiable, because it does not rely on standard variational methods. ${ }^{1}$ It can be also used when the designer is interested in efficiency maximizing, instead of revenue maximizing mechanisms. All the analysis goes through by replacing virtual utilities with actual utilities.

\footnotetext{
${ }^{*}$ We are grateful to Roger Myerson for a thought-provoking discussion on the phenomenon presented in this paper.

† Centro de Economía Aplicada, Universidad de Chile, República 701, Santiago, Chile, nicolasf@dii.uchile.cl.

${ }^{\ddagger}$ Leonard Stern School of Business, Kaufman Management Center, 44 West 4 th Street, KMC 7-64, New York, NY 10012,USA,vskreta@stern.nyu.edu.

${ }^{1}$ See for instance Lollivier and Rochet (1983).
} 
The fact that randomizations are a feature of revenue-maximizing mechanisms can be viewed as quite surprising given that the buyer is risk neutral and his type is single dimensional. In the continuum varieties model of Maskin and Riley (1989) these authors devote Section 5 to illustrate why randomizations are not a feature of revenue-maximizing auctions. Also Thanassoulis (2004) stresses that the randomizations in his environment are due to the fact that types are multidimensional. Why then do they appear in our environment? The reason is that we are considering a finite number of different products, and we allow for utilities to be non-linear in types. The feature of randomization is absent in the extreme cases of one, and a continuum of identical goods, but it can appear in intermediate cases where there is some discreteness in the number of goods and goods are heterogeneous.

\section{Example}

Suppose that there is a single buyer whose type, $v$, is distributed uniformly on the interval $[0,1]$ and that there are two possible allocations, $z_{1}$ and $z_{2}$. The buyer's payoffs from these two allocations are given by

$$
u^{z_{1}}(v)=0.5 e^{v}+0.524 \text { and } u^{z_{2}}(v)=e^{0.5 v}
$$

which are both convex and increasing in $v$. We let $Z=\left\{z_{1}, z_{2}\right\}$.

Our objective is to find the allocation mechanism that maximizes the seller's revenue. As usual, we can appeal to the revelation principle and search among the direct revelation mechanisms $(D R M)$ that satisfy truth-telling and voluntary participation. A $D R M$ here consists of an assignment rule and a payment rule $(p, x)$. For all $v p(v)=\left(p^{z_{1}}(v), p^{z_{2}}(v)\right)$ specifies the probability with which each allocation prevails and $x(v)$ is the expected payment incurred by the buyer.

Given a $\operatorname{DRM}(p, x)$ the buyer's maximized payoff is given by

$$
U(v) \equiv \max _{v^{\prime}} \sum_{z \in Z} p^{z}\left(v^{\prime}\right) u^{z}(v)-x\left(v^{\prime}\right)
$$

and it is convex, since it is a maximum of convex functions.

A $\operatorname{DRM}(p, x)$ is feasible if it satisfies (i) resource constraints, (ii) incentive compatibility constraints and (iii) voluntary participation. ${ }^{2}$

Resource constraints require that, for all $v$, we have that $0 \leq p^{z}(v) \leq 1$ and $\Sigma_{z \in Z} p^{z}(v)=1$. Necessary and sufficient conditions for incentive compatibility are that (a) the derivative of $U$ (more precisely a selection from its subgradient, which is single valued almost surely) evaluated at the true type, that is $P(v) \equiv \sum_{z \in Z} p^{z}(v) \frac{d u^{z}(v)}{d v}$, is weakly increasing, and (b) $U(v)=U(0)+\int_{0}^{v} P(s) d s$ for all $v \in V$. Participation constraints require that $U(v) \geq 0$.

\footnotetext{
${ }^{2}$ More details about the general results and the solution approach used in this example can be found in Figueroa and Skreta (2007).
} 
Below we use the following notation $P^{z_{i}}(v) \equiv \frac{d u^{z_{i}}(v)}{d v}$ for $i=1,2$. Then,

$$
P(v)=P^{z_{i}}(v) \text { when } p^{z_{i}}(v)=1 .
$$

We denote by $J_{z}(v)$ the virtual utility of allocation $z .^{3}$ For this example, virtual utilities are given by

$$
J_{z_{1}}(v)=0.5 v e^{v}+0.524 \text { and } J_{z_{2}}(v) \equiv e^{0.5 v}(0.5+0.5 v)
$$

and, as can be seen from Figure 1, they are strictly increasing in $v$.

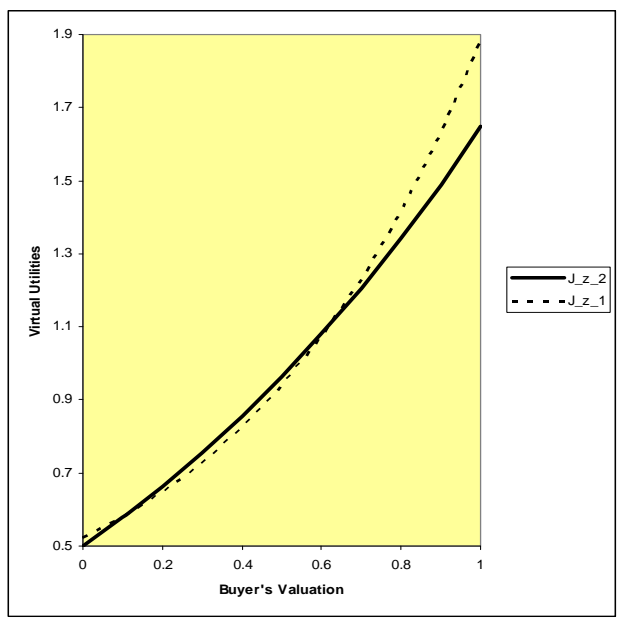

Figure 1

The graphs of $J_{z_{1}}$ and $J_{z_{2}}$ cross at two points $v^{*}=0.1054$ and $\hat{v}=0.6346$.

By using standard arguments, we can write the seller's problem as:

$$
\max _{(p, x)} \int_{0}^{1}\left[p^{z_{1}}(v) J_{z_{1}}(v)+p^{z_{2}}(v) J_{z_{2}}(v)\right] d v-U(0)
$$

subject to : $P(v)$ increasing and

$$
0 \leq p^{z_{i}}(v) \leq 1, i=0,1,2 \text { and } \sum_{i=0}^{2} p^{z_{i}}(v)=1
$$

However, in this example despite the fact that the virtual utilities of both allocations $z_{1}$ and $z_{2}$ are strictly increasing in $v$, pointwise optimization of (2) does not lead to a feasible mechanism.

\footnotetext{
${ }^{3}$ In general, the virtual utility of allocation $z$ is defined as$$
J_{z}(v) \equiv u^{z}(v)-\frac{[1-F(v)]}{f(v)} \frac{d u^{z}(v)}{d v} .
$$ 
As we can see from Figure 1, pointwise optimization dictates $p^{z_{1}}(v)=1$ for $v \in[0,0.1054]$ and $p^{z_{2}}(v)=1$ for $v$ in the interval $[0.1054,0.6346]$. However it is not possible to switch from allocation $z_{1}$ to allocation $z_{2}$, because this would imply that $P$ falls at 0.1054 (recall (1)), since as can be seen in Figure 2, $P^{z_{1}}(v)>P^{z_{2}}(v)$ for all $v \in(0,1]$.

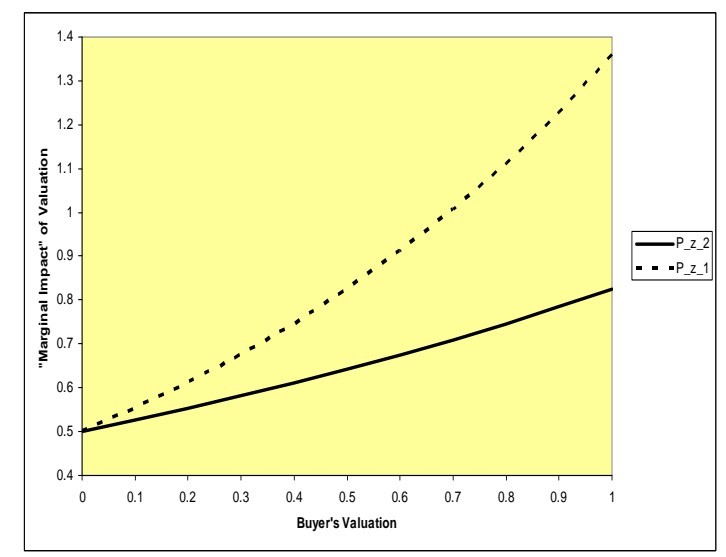

Figure 2

How should the seller proceed? At a solution the seller should mix between $z_{1}$ and $z_{2}$ in a way that minimizes the "cost" of having to choose with positive probability an allocation that does not have the highest virtual utility, subject to respecting the requirement that $P$ is increasing in $v$.

The region of "compromise" is an interval of the form $[\underline{x}, \bar{x}]$, where $\underline{x}$ and $\bar{x}$ satisfy $0 \leq \underline{x} \leq v^{*} \leq$ $\bar{x} \leq \hat{v}$, where $\hat{v}$ is the first point to the right of $v^{*}$ where $J_{z_{1}}$ and $J_{z_{2}}$ cross again. ${ }^{4}$ The loss of assigning positive weight to allocation $z_{2}$ for $v \in\left[\underline{x}, v^{*}\right)$ is given by $\int_{\underline{x}}^{v^{*}} p^{z_{2}}(v)\left[J_{z_{1}}(v)-J_{z_{2}}(v)\right] d v$ and the loss of assigning positive weight to allocation $z_{1}$ for $v \in\left[\underline{x}, v^{*}\right)$ is given by $\int_{v^{*}}^{\bar{x}} p^{z_{1}}(v)\left[J_{z_{2}}(v)-J_{z_{1}}(v)\right] d v$. An optimal mechanism must randomize between $z_{1}$ and $z_{2}$ on $[\underline{x}, \bar{x}]$, in a way such that the loss is minimized. Moreover $\underline{x}$ and $\bar{x}$ must be chosen optimally.

The problem to be solved is called Program A and it is given by: ${ }^{5}$

$$
\min _{p^{z_{1}, \underline{x}, \bar{x}}} \int_{\underline{x}}^{v^{*}}\left(1-p^{z_{1}}(v)\right)\left[J_{z_{1}}(v)-J_{z_{2}}(v)\right] d v+\int_{v^{*}}^{\bar{x}} p^{z_{1}}(v)\left[J_{z_{2}}(v)-J_{z_{1}}(v)\right] d v .
$$

subject to:

(i) $P(v) \equiv p^{z_{1}}(v) \frac{d u^{z_{1}}(v)}{d v}+\left(1-p^{z_{1}}(v)\right) \frac{d u^{z_{2}}(v)}{d v}$ increasing in $v$ for $v \in[\underline{x}, \bar{x}]$

(ii) if $\underline{x}>0$, then $p^{z_{1}}(\underline{x})=1$ (and $P(\underline{x})=P^{z_{1}}(\underline{x})$ ) and if $\bar{x}<\hat{v}$ then $p^{z_{2}}(\bar{x})=1$ (and $\left.P(\bar{x})=P^{z_{2}}(\bar{x})\right)$

(iii) $0 \leq \underline{x} \leq v^{*} \leq \bar{x} \leq \hat{v}$

\footnotetext{
${ }^{4}$ Such a point exists, since $J_{z_{i}}(1)=u^{z_{i}}(1)$, and we have that $\frac{d u^{z_{1}}(v)}{d v}>\frac{d u^{z_{2}}(v)}{d v}$, so $J_{z_{1}}(1)>J_{z_{2}}(1)$ unless $u^{z_{2}}(0)>u^{z_{1}}(0)$. This last situation is not possible, since then we would not have had the first crossing.

${ }^{5}$ Since for each $v$ it must be that $p^{z_{1}}(v)+p^{z_{2}}(v)=1$, we will express $p^{z_{2}}(v)$ as $1-p^{z_{1}}(v)$.
} 
The constraints (i) and (ii) guarantee that $P$ is increasing and thus the solution is incentive compatible. Constraints in (ii) arise because outside the region of "compromise" $[\underline{x}, \bar{x}]$ the best for the seller is to assign probability one to $z_{1}$ for $v \in[0, \underline{x}]$ and probability one to allocation $z_{2}$ for $v \in[\bar{x}, \hat{v}]$.

Now we establish that Program A is equivalent to a much simpler problem where the only choice variable is $\underline{x}$. This is done with the help of a couple of results.

Our first result states that an optimal assignment rule randomizes between allocation $z_{1}$ and allocation $z_{2}$ in such a way, that $P$ remains constant over $[\underline{x}, \bar{x}]$. Its proof is straightforward and we omit it. The interested reader is referred to the working paper version of this work.

Lemma 1 An optimal assignment rule randomizes between allocations $z_{1}$ and $z_{2}$ over an interval $[\underline{x}, \bar{x}]$, with $0 \leq \underline{x} \leq \bar{x} \leq \hat{v}$, in a way such that

$$
P(v) \equiv p^{z_{1}}(\underline{x}) \frac{d u^{z_{1}}(\underline{x})}{d v}+\left(1-p^{z_{1}}(\underline{x})\right) \frac{d u^{z_{2}}(\underline{x})}{d v} \equiv P(\underline{x}) .
$$

The intuition for this result is simple. Optimality dictates that $P$ is as small as possible to the right of $v^{*}$, where $z_{2}$ is preferred by the seller, and $P$ is as large as possible to the left of $v^{*}$, where the seller prefers $z_{1}$. Since $P$ must be increasing these two forces imply that for the interval where the seller is mixing $P$ must be flat. Put in another way, since the monotonicity constraint is binding, optimality dictates that it is satisfied with "equality", so $P$ is flat and not strictly increasing.

Next, observe that $\bar{x}$ can be pinned down by $\underline{x}$ and $p^{z_{1}}$. It is either the smallest $v$ where $p^{z_{1}}(v)=0$, or if such a $v$ does not exist, it is equal to 1 . That is

$$
\bar{x}=\min \left\{\hat{v}, \min \left\{v \in[\underline{x}, \hat{v}] \mid p^{z_{1}}(v)=0\right\}\right\} .^{6}
$$

Our next result shows that at an optimum if $\underline{x}>0$, then the seller assigns probability one to $z_{1}$ at $\underline{x}$, whereas if $\underline{x}=0$, then she assigns probability one to $z_{2}$.

Lemma 2 If at an optimum $\underline{x}>0$, then $p^{z_{1}}(\underline{x})=1$, whereas, if at an optimum $\underline{x}=0$, then $p^{z_{1}}(\underline{x})=0$.

Proof. See Appendix.

If $\underline{x}>0$, Lemma 2 implies that $p^{z_{1}}(\underline{x})=1$ and with the help of (4) we get

$$
p^{z_{1}}(v) \frac{d u^{z_{1}}(v)}{d v}+\left(1-p^{z_{1}}(v)\right) \frac{d u^{z_{2}}(v)}{d v}=\frac{d u^{z_{1}}(\underline{x})}{d v}
$$

which implies that at a solution, when $\underline{x}>0$ then

$$
p^{z_{1}}(v)=\frac{\frac{d u^{z_{1}}(\underline{x})}{d v}-\frac{d u^{z_{2}}(v)}{d v}}{\frac{d u^{z_{1}(v)}}{d v}-\frac{d u^{z_{2}(v)}}{d v}} .
$$

\footnotetext{
${ }^{6}$ Recall that if $\bar{x}<\hat{v}$ then $p^{z_{2}}(\bar{x})=1$.
} 
Now, if $\underline{x}=0$, Lemma 2 tells us that $p^{z_{1}}(0)=0$, which is equivalent to $p^{z_{2}}(0)=1$, which immediately implies that $0=\underline{x}=\bar{x}$, and therefore

$$
p^{z_{2}}(v)=1 \text { for all } v \in[0, \hat{v}] .
$$

From (6) and (7) one can see that Program A can be stated as a problem where the control variable is simply $\underline{x}$. We call this problem Program B:

$$
\min _{\underline{x} \in\left[0, v^{*}\right]} \int_{\underline{x}}^{v^{*}}\left(1-p^{z_{1}}(v)\right)\left[J_{z_{1}}(v)-J_{z_{2}}(v)\right] d v+\int_{v^{*}}^{\bar{x}(\underline{x})} p^{z_{1}}(v)\left[J_{z_{2}}(v)-J_{z_{1}}(v)\right] d v,
$$

where $\bar{x}(\underline{x})$ satisfies $(5)$ and $p^{z_{1}}(\underline{x})$ satisfies either (6) or (7).

If a solution of Program B is interior, that is, if $\underline{x}>0$, then, an optimal allocation rule is given by

$$
\begin{aligned}
& p^{*}(v)=\left(p^{z_{1}}(v), p^{z_{2}}(v)\right)=(1,0) \text { for } v \in[0, \underline{x}) \\
& p^{*}(v)=\left(\frac{\frac{d u^{z_{1}}(\underline{x})}{d v}-\frac{d u^{z_{2}}(v)}{d v}}{\frac{d u^{z_{1}(v)}}{d v}-\frac{d u^{z_{2}(v)}}{d v}}, 1-\frac{\frac{d u^{z_{1}}(\underline{x})}{d v}-\frac{d u^{z_{2}}(v)}{d v}}{\frac{d u^{z_{1}(v)}}{d v}-\frac{d u^{z}(v)}{d v}}\right) \text { for }[\underline{x}, \bar{x}] \\
& p^{*}(v)=\left(p^{z_{1}}(v), p^{z_{2}}(v)\right)=(0,1) \text { for } v \in(\bar{x}, \hat{v}],
\end{aligned}
$$

otherwise, that is, if $\underline{x}=0$, then $p^{*}$ is $p^{*}(v)=(0,1)$ for $v \in[0, \hat{v}]$. The payment rule can be obtained from the allocation rule exactly as in Myerson (1981).

For our example, (8) becomes:

$$
\begin{aligned}
& \int_{\underline{x}}^{0.1054} \frac{e^{\underline{x}}-e^{v}}{e^{0.5 v}-e^{v}}\left[0.5 v e^{v}-e^{0.5 v}(0.5 v+0.5)+0.524\right] d v \\
& +\int_{0.1054}^{2 \underline{x}} \frac{e^{0.5 v}-e^{\underline{x}}}{e^{0.5 v}-e^{v}}\left[e^{0.5 v}(0.5 v+0.5)-0.5 v e^{v}-0.524\right] d v
\end{aligned}
$$


which is depicted in Figure 3.

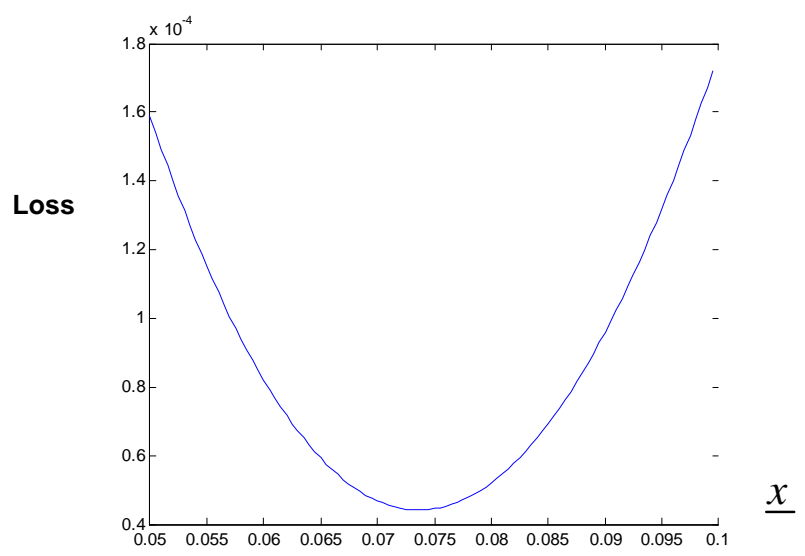

Figure 3: The Loss Function

It has a unique minimizer ${ }^{7}$ at

$$
\underline{x}=0.074,
$$

which with the help of (5) and (6) implies

$$
p^{z_{1}}(v)=\frac{e^{0.5 v}-e^{0.074}}{e^{0.5 v}-e^{v}} \text { and } \bar{x}=0.148 .
$$

Substituting (10) and (11) in (9) we get that an optimal assignment rule is

$$
\begin{aligned}
& p^{*}(v)=(1,0) \text { for } v \in[0,0.074) \\
& p^{*}(v)=\left(\frac{e^{0.5 v}-e^{0.074}}{e^{0.5 v}-e^{v}}, 1-\frac{e^{0.5 v}-e^{0.074}}{e^{0.5 v}-e^{v}}\right) \text { for } v \in[0.074,0.148) \\
& p^{*}(v)=(0,1) \text { for } v \in[0.148,0.6346] \\
& p^{*}(v)=(1,0) \text { for } v \in(0.6346,1]
\end{aligned}
$$

which is incentive compatible by construction. Figure 4 depicts the probability of $z_{1}$ that $p^{*}$ assigns around the region of randomization.

\footnotetext{
${ }^{7}$ Calculations and graphs for this example have been done with Matlab. Code available upon request.
} 


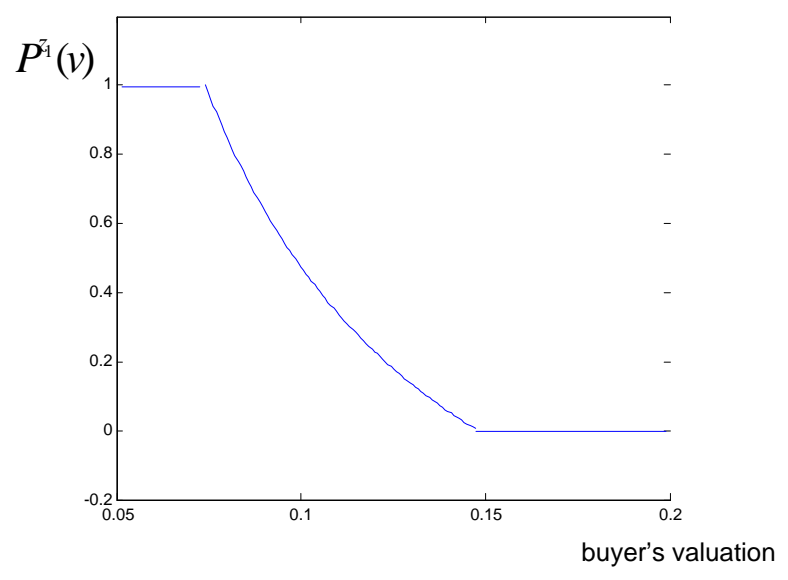

Figure 4: Optimal Randomization

In this example the optimal assignment rule involves randomizations. This is in contrast to the classical case, where (excluding cases where the seller is indifferent) an optimal allocation rule is deterministic.

In general, when there are more allocations, and/or when virtual utilities cross many times, and/or when $P^{z \prime} s$ are not always ranked in the same way, the details of the solution depend on the particular specifics of the problem at hand. However, the main idea of how to proceed is the one we illustrated. Whenever there is a point where $I C$ is violated by the assignment rule obtained via pointwise optimization, a solution involves an interval of randomization between more than one allocations. Of course, it is possible that in some cases this interval is degenerate.

\section{Appendix}

\section{Proof of Lemma 2}

Recall from (4) that all $v \in[\underline{x}, \bar{x}]$ we have that

$$
p^{z_{1}}(v) \frac{d u^{z_{1}}(v)}{d v}+\left(1-p^{z_{1}}(v)\right) \frac{d u^{z_{2}}(v)}{d v}=\left.\left[p^{z_{1}}(v) \frac{d u^{z_{1}}(v)}{d v}+\left(1-p^{z_{1}}(v)\right) \frac{d u^{z_{2}}(v)}{d v}\right]\right|_{\underline{x}} .
$$

Now from (13) we can obtain that

$$
p^{z_{1}}(v)=\frac{\left.p^{z_{1}}(\underline{x}) \frac{d u^{z_{1}}(v)}{d v}\right|_{\underline{x}}+\left.\left(1-p^{z_{1}}(\underline{x})\right) \frac{d u^{z_{2}}(v)}{d v}\right|_{\underline{x}}-\frac{d u^{z_{2}}(v)}{d v}}{\frac{d u^{z_{1}(v)}}{d v}-\frac{d u^{z_{2}(v)}}{d v}}
$$

so the objective function can be written as 


$$
\begin{aligned}
& L\left(\underline{x}, p^{z_{1}}(\underline{x})\right)=\int_{\underline{x}}^{v^{*}}\left(1-\frac{\left[p^{z_{1}}(\underline{x}) \frac{d u^{z_{1}}(\underline{x})}{d v}+\left(1-p^{z_{1}}(\underline{x})\right) \frac{d u^{z_{2}(\underline{x})}}{d v}-\frac{d u^{z_{2}(v)}}{d v}\right]}{\frac{d u^{z_{1}(v)}}{d v}-\frac{d u^{z_{2}(v)}}{d v}}\right)\left[J_{z_{1}}(v)-J_{z_{2}}(v)\right] d v \\
& +\int_{v^{*}}^{\bar{x}\left(\underline{x}, p^{z_{1}}(\underline{x})\right)} \frac{p^{z_{1}}(\underline{x}) \frac{d u^{z_{1}}(\underline{x})}{d v}+\left(1-p^{z_{1}}(\underline{x})\right) \frac{d u^{z_{2}}(\underline{x})}{d v}-\frac{d u^{z_{2}}(v)}{d v}}{\frac{d u^{z_{1}(v)}}{d v}-\frac{d u^{z_{2}(v)}}{d v}}\left[J_{z_{2}}(v)-J_{z_{1}}(v)\right] d v .
\end{aligned}
$$

From constraints (ii) of the problem stated in (3) we have that if $\underline{x}>0$ then $p^{z_{1}}(\underline{x})=1$. This observation implies that depending on whether $\underline{x}$ is interior or not we have that

$$
L\left(\underline{x}, p^{z_{1}}(\underline{x})\right)=\left\{\begin{array}{ll}
L(\underline{x}, 1) & \text { if } \underline{x}>0 \\
L\left(0, p^{z_{1}}(0)\right) & \text { if } \underline{x}=0
\end{array} .\right.
$$

To find a minimum of $L$, we look separately in each region. First, we look at the interior region where $\underline{x}>0$ which implies that $p^{z_{1}}(\underline{x})$ must be 1 . Differentiating $L$ with respect to $\underline{x}$ we get

$$
\begin{aligned}
\frac{d L(\underline{x})}{d \underline{x}}= & {\left.\left[\frac{\left.\frac{d u^{z_{2}(v)}}{d v}\right|_{\underline{x}}-\frac{d u^{z_{2}}}{d v}}{\frac{d u^{z_{1}(v)}}{d v}-\frac{d u^{z_{2}(v)}}{d v}}\right]\right|_{\bar{x}(\underline{x})}\left[J_{z_{2}}(\bar{x}(\underline{x}))-J_{z_{1}}(\bar{x}(\underline{x}))\right] \frac{d \bar{x}(\underline{x})}{d \underline{x}} } \\
& +\int_{\underline{x}}^{\bar{x}(\underline{x})} \frac{\left.\frac{d^{2} u^{z_{1}}(v)}{d v^{2}}\right|_{\underline{x}}}{\frac{d u^{z_{1}(v)}}{d v}-\frac{d u^{z} z_{(v)}}{d v}}\left[J_{z_{2}}(v)-J_{z_{1}}(v)\right] d v .
\end{aligned}
$$

The first term is zero, because by the definition of $\bar{x}$ it follows that if $\bar{x}(\underline{x})<\hat{v}$, then

$$
\left.p^{z_{1}}(\bar{x}(\underline{x})) \equiv\left[\frac{\left.\frac{d u^{z_{2}(v)}}{d v}\right|_{\underline{x}}-\frac{d u^{z_{2}}}{d v}}{\frac{d u^{z_{1}(v)}}{d v}-\frac{d u^{z_{2}(v)}}{d v}}\right]\right|_{\bar{x}(\underline{x})}=0,
$$

whereas if $\bar{x}(\underline{x})=\hat{v}$, then $\frac{d \bar{x}(\underline{x})}{d \underline{x}}=0$. Then, it is easy to see that the sign of $\frac{d L(\underline{x})}{d \underline{x}}$ depends only on the sign of $\int_{\underline{x}}^{\bar{x}(\underline{x})} \frac{J_{z_{2}}(v)-J_{z_{1}}(v)}{\frac{d u^{2} 1(v)}{d v}-\frac{d u^{z} 2(v)}{d v}} d v$, since $\left.\frac{d^{2} u^{z}(v)}{d v^{2}}\right|_{\underline{x}}$ is positive by convexity. Moreover, $\int_{\underline{x}}^{\bar{x}(\underline{x})} \frac{J_{z_{2}}(v)-J_{z_{1}}(v)}{\frac{d u^{z} 1(v)}{d v}-\frac{d u^{z} 2(v)}{d v}} d v$ is (weakly) increasing in $\underline{x}$ : the integrand is negative when $v<v^{*}$ and positive otherwise, and $\bar{x}(\underline{x})$ is (weakly) increasing in $\underline{x}$.

This observation, plus the fact that $\left.\left[\frac{d u^{z_{1}}(v)}{d v}-\frac{d u^{z_{2}}(v)}{d v}\right]\right|_{\underline{x}=v^{*}}>0$ and $\int_{v^{*}}^{\bar{x}(0)} \frac{J_{z_{2}}(v)-J_{z_{1}}(v)}{\frac{d u^{2} 1(v)}{d v}-\frac{d u^{2}(v)}{d v}} d v>0$, imply that $\frac{d L(\underline{x})}{d \underline{x}}$ is either always positive, or that it crosses 0 at some point. Then, 


$$
\underline{x}= \begin{cases}0 & \text { if } \int_{0}^{\bar{x}(0)} \frac{J_{z_{2}}(v)-J_{z_{1}}(v)}{\frac{d u^{z} 1(v)}{d v}-\frac{d u^{z}(v)}{d v}} d v \geq 0 \\ {\left[\frac{d L(\underline{x})}{d \underline{x}}\right]^{-1}(0)} & \text { if not }\end{cases}
$$

which gives us an optimal $\underline{x}$ when we are constrained to an optimal starting mixture of $p^{z_{1}}(\underline{x})=1$, which must be the case when $\underline{x}$ is interior.

However, when $\underline{x}=0$ then it is possible to choose $p^{z_{1}}(0) \neq 1$. Fixing $\underline{x}=0$, the objective function becomes a function only of $p^{z_{1}}(0)$. Differentiating it and doing similar manipulations as before, we get

$$
\frac{d L\left(p^{z_{1}}(0)\right)}{d p^{z_{1}}(0)}=\left.\left[\frac{d u^{z_{1}}(v)}{d v}-\frac{d u^{z_{2}}(v)}{d v}\right]\right|_{\underline{x}} \int_{\underline{x}}^{\bar{x}(\underline{x})} \frac{J_{z_{2}}(v)-J_{z_{1}}(v)}{\frac{d u^{z_{1}}(v)}{d v}-\frac{d u^{z_{2}(v)}}{d v}} d v,
$$

which is independent of $p^{z_{1}}(\underline{x})$, hence we obtain that a minimizer is:

$$
p^{z_{1}}(0)=\left\{\begin{array}{cc}
0 & \text { if } \int_{0}^{\bar{x}(0)} \frac{J_{z_{2}}(v)-J_{z_{1}}(v)}{\frac{d u^{2} 1(v)}{d v}-\frac{d u^{2}(v)}{d v}} d v \geq 0 . \\
1 & \text { if not }
\end{array}\right.
$$

Therefore, by (14) and (15) we see that everything depends on the sign of $\int_{0}^{\bar{x}(0)} \frac{J_{z_{2}}(v)-J_{z_{1}}(v)}{\frac{d u^{2} 1(v)}{d v}-\frac{d u^{2} 2(v)}{d v}} d v$. If negative, then the optimal $\underline{x}$ is interior, and $p^{z_{1}}(\underline{x})=1$, for $\underline{x}>0$. If positive, then $\underline{x}=0$ dominates any other starting point, and $p^{z_{1}}(0)=0$, for $\underline{x}=0$.

\section{REFERENCES}

[1] Lollivier, S., and J.-C. Rochet (1983): "Bunching and Second-order Conditions: A Note on Optimal Tax Theory," Journal of Economic Theory 31, 392-400.

[2] Figueroa, N. and V. Skreta (2007): "A Note on Optimal Allocation Mechanisms," working paper.

[3] Maskin, E. and J. Riley (1989): "Optimal Multi-Unit Auctions," in The Economics of Missing Markets, ed. by F. Hahn, 312-335.

[4] Myerson, (1981): "Optimal Auction Design," Mathematics of Operations Research, 6, 58-73.

[5] Riley, J. G. and W. F. Samuelson (1981): "Optimal Auctions," American Economic Review, 71, 381-392.

[6] Thanassoulis, J. (2004): "Haggling over Substitutes," Journal of Economic Theory, 117, 217245. 